PROCEEDINGS OF THE

AMERICAN MATHEMATICAL SOCIETY

Volume 129, Number 6, Pages 1843-1851

S 0002-9939(00)05851-2

Article electronically published on November 3, 2000

\title{
GEODESICS ON THE SPACE OF LAGRANGIAN SUBMANIFOLDS IN COTANGENT BUNDLES
}

\author{
DARKO MILINKOVIĆ
}

(Communicated by Jozef Dodziuk)

\begin{abstract}
We prove that the space of Hamiltonian deformations of zero section in a cotangent bundle of a compact manifold is locally flat in the Hofer metric and we describe its geodesics.
\end{abstract}

\section{INTRODUCTION}

Let $M$ be a compact smooth manifold. Denote by $\mathcal{L}(M)$ the space of Hamiltonian deformations of zero section $O_{M}$ in its cotangent bundle $T^{*} M$. For $L_{0} \in \mathcal{L}(M)$ and for a path $L_{t}:=\phi_{t}^{H}\left(L_{0}\right)$, where $\phi_{t}^{H}$ is a Hamiltonian isotopy generated by a smooth compactly supported Hamiltonian function $H:[0,1] \times T^{*} M \rightarrow \mathbf{R}$, we define

$$
\text { length }\left(\left\{L_{t}\right\}\right):=\inf \int_{0}^{1}\left(\max _{x} H(t, x)-\min _{x} H(t, x)\right) d t,
$$

where the infimum is taken over all $H$ such that $\phi_{t}^{H}\left(L_{0}\right)=L_{t}$. For $L_{1} \in \mathcal{L}(M)$, define distance between $L_{0}$ and $L_{1}$ as the infimum of lengths over connecting paths. More precisely,

$$
d\left(L_{0}, L_{1}\right):=\inf _{H}\left\{\int_{0}^{1}\left(\max _{x} H(t, x)-\min _{x} H(t, x)\right) d t \mid \phi_{1}^{H}\left(L_{0}\right)=L_{1}\right\} .
$$

Note that $\left\{L_{t}\right\}$ depends only on values of $H(t, x)$ for $x$ near $\bigcup_{t} L_{t}$. Since the distance defined by (2) is the infimum of lengths over all connecting paths, $d$ does not depend on whether the maximum and the minimum are taken over $x \in \bigcup_{t} L_{t}$ or over $T^{*} M$. In this paper $\max _{x}$ and $\min _{x}$ will denote the maximum and minimum over $x \in \bigcup_{t} L_{t}$.

A group of Hamiltonian diffeomorphisms of $T^{*} M$ acts on $\mathcal{L}(M)$ via $(\psi, L) \mapsto$ $\psi(L)$. It is known that $d$ is an invariant distance on $\mathcal{L}(M)$ with respect to this action. The most delicate fact in the proof of this fact is the non-degeneracy of $d$ (see [2], [8]). A proof given by Oh 8 is based on a study of invariants defined by

$$
\rho(H)=\inf \left\{\lambda \mid H F_{*}^{(-\infty, \lambda)}(H) \rightarrow H F_{*}(H) \text { is surjective }\right\},
$$

where $H F_{*}$ is Floer homology. After a certain normalization, $\rho(H)$ depends only on $L=\phi_{1}^{H}\left(O_{M}\right)$ and it is denoted by $\rho(L)$ (see 8 . or Section 2 below for more details).

Received by the editors September 21, 1999.

1991 Mathematics Subject Classification. Primary 58E05; Secondary 57R57, 58F05.

(C)2000 American Mathematical Society 
In this paper we prove that every $L \in \mathcal{L}(M)$ has a flat $C^{1}$-neighborhood. More precisely, let $\mathcal{G}$ be the $C^{1}$-neighborhood (in the space of Lagrangian embeddings) of $O_{M}$ such that if $L \in \mathcal{G}$, then $L=G \operatorname{raph}(d S)$ for some smooth function $S$ on $M$. We prove that for $L_{i}=\operatorname{Graph}\left(d S_{i}\right) \in \mathcal{G}, i \in\{0,1\}$,

$$
d\left(L_{0}, L_{1}\right)=\left\|S_{1}-S_{0}\right\|:=\max \left(S_{1}-S_{0}\right)-\min \left(S_{1}-S_{0}\right) .
$$

As a corollary, we obtain a description of geodesics on $\mathcal{L}(M)$. This generalizes an analogous result by Bialy and Polterovich for Hamiltonian diffeomorphisms in $\mathbf{R}^{2 n}$ [1. A description of geodesics on the group of Hamiltonian diffeomorphisms in general symplectic manifolds is obtained by Lalonde and McDuff [ 5 .

We would like to thank the referee for several valuable corrections and suggestions.

\section{Preliminaries}

Let $\left\{L_{t}\right\}_{0 \leq t \leq 1}$ be a smooth regular path in $\mathcal{L}$, i.e. $\frac{d}{d t} L_{t} \neq 0$ for every $t \in[0,1]$. $L_{t}$ is called a minimal geodesic if length $\left(\left\{L_{t}\right\}\right)=d\left(L_{0}, L_{1}\right)$. It is called geodesic if it is a minimal geodesic locally on $[0,1]$ (compare [1]).

A Hamiltonian $H(t, x)$ is called quasi-autonomous if there exist $x_{+}, x_{-} \in \bigcup_{t} L_{t}$ such that $\max _{x} H(t, x)=H\left(t, x_{+}\right)$and $\min _{x} H(t, x)=H\left(t, x_{-}\right)$for every $t$. This is equivalent to

$$
\int_{0}^{1}\left(\max _{x} H(t, x)-\min _{x} H(t, x)\right) d t=\max _{x} \int_{0}^{1} H(t, x) d t-\min _{x} \int_{0}^{1} H(t, x) d t
$$

(see [1]).

Let us recall a construction of symplectic invariants by $\mathrm{Oh}[8]$. For $H \in$ $C_{0}^{\infty}\left([0,1] \times T^{*} M\right)$ consider the classical action functional

$$
\mathcal{A}_{H}(\gamma):=\int_{0}^{1} \gamma^{*} p d q-H d t
$$

for $\gamma:[0,1] \rightarrow T^{*} M, \gamma(0) \in O_{M}$, where $p d q$ is a canonical Liouville 1-form on $T^{*} M$. Floer chain complexes $C F_{*}(H)$ are defined as free $\mathbf{Z}$-modules over

$$
\operatorname{Crit}_{*}\left(\mathcal{A}_{H}\right):=\left\{\gamma:[0,1] \rightarrow T^{*} M \mid \frac{d \gamma}{d t}=X_{H}(\gamma), \gamma(0), \gamma(1) \in O_{M}\right\}
$$

where $X_{H}$ is a Hamiltonian vector field corresponding to $H$. They are graded by the Maslov index, and filtered by level sets of $\mathcal{A}_{H}: C F_{*}^{\lambda}(H)$ denotes a free $\mathbf{Z}$-module over

$$
\operatorname{Crit}_{*}\left(\mathcal{A}_{H}\right):=\left\{\gamma \in C F_{*}(H) \mid \mathcal{A}_{H}(\gamma) \leq \lambda\right\} .
$$

Recall that $H F_{*}(H)$ is defined as a homology group of $C F_{*}(H)$ with respect to the boundary homomorphism

$$
\partial: C F_{*}(H) \rightarrow C F_{*}(H), \partial(x):=\sum_{y \in C F(H)} n(x, y) y,
$$

where $n(x, y)$ is the number of solutions of

$$
\left\{\begin{array}{l}
\frac{\partial u}{\partial \tau}+J\left(\frac{\partial u}{\partial t}-X_{H}(u)\right)=0, \\
u(\tau, 0), u(\tau, 1) \in O_{M}, \\
u(-\infty, t)=x(t), u(+\infty, t)=y(t)
\end{array}\right.
$$

("negative gradient flow of $\mathcal{A}_{H}$ "). Here $J$ is some almost complex structure compatible with the symplectic form. Since $\mathcal{A}_{H}$ decreases along its "negative gradient 
lines" (21), $\partial$ restricts to $C F_{*}^{\lambda}(H)$; the corresponding homology group is denoted by $H F_{*}^{\lambda}(H)$. An obvious inclusion $C F_{*}^{\lambda}(H) \rightarrow C F_{*}(H)$ induces a homomorphism

$$
j_{*}^{\lambda}: H F_{*}^{\lambda}(H) \rightarrow H F_{*}(H) .
$$

Oh [8] defined for a generic $H \in C_{0}^{\infty}\left([0,1] \times T^{*} M\right)$

$$
\rho(H)=\inf \left\{\lambda \mid j_{*}^{\lambda}: H F^{\lambda}(H)_{*} \rightarrow H F_{*}(H) \text { is surjective }\right\},
$$

and proved that it depends only on $L:=\phi_{1}^{H}\left(O_{M}\right)$, but not on a particular choice of normalized $H \in C_{0}^{\infty}\left([0,1] \times T^{*} M\right)$ that generates $L$. More precisely, denote by $W_{H}$ the wave front of $H$, i.e.

$$
W_{H}:=\left\{(q, s) \in M \times \mathbf{R} \mid q=\pi(x), s=\mathcal{A}_{H}\left(\phi_{t}^{H} \circ\left(\phi_{1}^{H}\right)^{-1}(x)\right), x \in L\right\},
$$

where $\pi: T^{*} M \rightarrow M$ is the canonical projection. Then, if $\phi_{1}^{H}\left(O_{M}\right)=\phi_{1}^{K}\left(O_{M}\right)$ and $W_{H}=W_{K}, \rho(H)=\rho(K)$ (Theorem 8.1 in [8]).

Let $\mathcal{H}(M)$ be a set of Hamiltonians normalized so that their wave fronts depend only on $L:=\phi_{1}^{H}\left(O_{M}\right)$ :

$$
\mathcal{H}(M):=\left\{H \in C_{0}^{\infty}\left([0,1] \times T^{*} M\right) \mid \max _{(x, s) \in W_{H}} s+\min _{(x, s) \in W_{H}} s=0\right\} .
$$

Note that definitions (1) and (2) remain the same if we take the infimum over $H \in \mathcal{H}(M)$ only. Indeed, it is clear that

$$
\begin{aligned}
& \inf _{H \in C_{0}^{\infty}\left([0,1] \times T^{*} M\right)}\left\{\int_{0}^{1}\left(\max _{x} H(t, x)-\min _{x} H(t, x)\right) d t \mid \phi_{t}^{H}\left(0_{M}\right)=L_{t}\right\} \\
& \leq \inf _{H \in \mathcal{H}(M)}\left\{\int_{0}^{1}\left(\max _{x} H(t, x)-\min _{x} H(t, x)\right) d t \mid \phi_{t}^{H}\left(0_{M}\right)=L_{t}\right\} .
\end{aligned}
$$

For every $H \in C_{0}^{\infty}\left([0,1] \times T^{*} M\right)$ there exist $c_{0} \in \mathbf{R}$ and $\chi \in C_{0}^{\infty}\left(T^{*} M\right)$ such that $\chi=1$ in a neighborhood of $\bigcup_{t} \operatorname{supp} H(t, \cdot), \chi \leq 1$, and $H^{c_{0}, \chi}:=\left(H+c_{0}\right) \chi \in \mathcal{H}(M)$. Since $H^{c_{0}, \chi}$ generates $L_{t}$ and

$$
\max _{x} H^{c_{0}, \chi}(t, x)-\min _{x} H^{c_{0}, \chi}(t, x) \leq \max _{x} H(t, x)-\min _{x} H(t, x),
$$

it follows that (4) is an equality.

Recall that for generic $H, K \in C_{0}^{\infty}\left([0,1] \times T^{*} M\right)$ the homomorphism $h_{*}$ : $H F_{*}(H) \rightarrow H F_{*}(K)$ is induced by the homomorphism defined on the chain level as

$$
h: C F_{*}(H) \rightarrow C F_{*}(K), h(x):=\sum_{y \in C F(K)} n(x, y) y,
$$

where $n(x, y)$ is the number of solutions of

$$
\left\{\begin{array}{l}
\frac{\partial u}{\partial \tau}+J\left(\frac{\partial u}{\partial t}-X_{\tilde{H}}(u)\right)=0 \\
u(\tau, 0), u(\tau, 1) \in O_{M}, \\
u(-\infty, t)=x(t), u(+\infty, t)=y(t) .
\end{array}\right.
$$

Here $\tilde{H}(\tau, t, x)$ is a generic path in $C_{0}^{\infty}\left([0,1] \times T^{*} M\right)$, such that $\tilde{H}(\tau, t, x)=H(t, x)$ for $\tau \leq-1$ and $\tilde{H}(\tau, t, x)=K(t, x)$ for $\tau \geq 1$. Since $\mathcal{A}_{H}$ decreases along its "negative gradient lines" (2), it follows that $j_{*}^{\lambda}$ commutes with $h_{*}$. This fact, together with careful analysis of change of $\mathcal{A}_{H}$ along the trajectories (2) makes it possible to express effects of the homomorphism $h_{*}$ on the level sets of the action functional and to prove that $\rho$ is $C^{0}$ continuous. This extends the definition of $\rho$ 
to all (not necessarily generic) $H \in \mathcal{H}(M)$ (we refer the reader to [8] for details). Main properties of $\rho$ are summarized in the following

Proposition 1. The function $\rho: \mathcal{H}(M) \rightarrow \mathbf{R}$ satisfies

1. If $L:=\phi_{1}^{H}\left(O_{M}\right)=\phi_{1}^{K}\left(O_{M}\right)$, then $\rho(H)=\rho(K)$; hence we can denote $\rho(H)$ by $\rho(L)$.

2. $\rho(L) \in \operatorname{Spec}(L):=\left\{\mathcal{A}_{H}\left(\phi_{t}^{H} \circ\left(\phi_{1}^{H}\right)^{-1}(x)\right) \mid x \in O_{M} \cap L\right\}$

3 .

$$
\begin{aligned}
-\int_{0}^{1} \max _{x \in T^{*} M}(H(t, x)-K(t, x)) d t & \leq \rho(H)-\rho(K) \\
& \leq-\int_{0}^{1} \min _{x \in T^{*} M}(H(t, x)-K(t, x)) d t .
\end{aligned}
$$

In particular, $\rho$ is $C^{0}$-continuous and monotone, i.e. if $K \leq H$, then $\rho(H) \leq$ $\rho(K)$.

4. $\rho(0)=0$.

5. $\rho\left(\phi_{1}^{H}\left(O_{M}\right)\right)+\rho\left(\left(\phi_{1}^{H}\right)^{-1}\left(O_{M}\right)\right) \leq d\left(O_{M}, \phi_{1}^{H}\left(O_{M}\right)\right)$.

6. If $S: M \rightarrow \mathbf{R}$ is a smooth function, then $\rho\left(-\pi^{*} S\right)=\max S$, where $\pi$ : $T^{*} M \rightarrow M$ is a canonical projection.

Proof. 1. is the contents of Theorem 8.1 in [8]. 2.-4. are contained in Theorem II in [8]. From 3. and 4. follows

$$
\rho\left(\phi_{1}^{H}\left(O_{M}\right)\right) \leq-\int_{0}^{1} \min _{x \in T^{*} M} H(t, x) d t .
$$

Since $\left(\phi_{1}^{H}\right)^{-1}=\phi_{1}^{\bar{H}}$, where $\bar{H}(t, x)=-H\left(t, \phi_{t}^{H}(x)\right)$, (5) gives

$$
\begin{aligned}
\rho\left(\left(\phi_{1}^{H}\right)^{-1}\left(O_{M}\right)\right) & \leq-\int_{0}^{1} \min _{x \in T^{*} M} \bar{H}(t, x) d t \\
& =-\int_{0}^{1} \min _{x \in T^{*} M}\{-H(t, x)\} d t \\
& =\int_{0}^{1} \max _{x \in T^{*} M} H(t, x) d t .
\end{aligned}
$$

Adding (5) and (6) we get

$$
\rho\left(\phi_{1}^{H}\left(O_{M}\right)\right)+\rho\left(\left(\phi_{1}^{H}\right)^{-1}\left(O_{M}\right)\right) \leq \operatorname{length}\left(\left\{\phi_{t}^{H}\left(O_{M}\right)\right\}\right) .
$$

Taking $\inf _{H}$ we get 5 .

To prove 6., note that $C F_{*}\left(\pi^{*} S\right)$ consists of constant paths (critical points of $S$ ), and thus for $x \in C F_{*}\left(-\pi^{*} S\right)$

$$
\mathcal{A}_{-\pi^{*} S}(x)=S(x) .
$$

Let $x_{+} \in M$ be such that $S\left(x_{+}\right)=\max S$. By the non-triviality of the cap action (see [3], or 9], 6] for similar arguments) for a generic $x \in M$ there exists a trajectory (2) connecting the generators of $C F_{\operatorname{dim} M}\left(-\pi^{*} S\right)$ and $C F_{0}\left(-\pi^{*} S\right)$ that contribute to the generators of $H F_{\operatorname{dim} M}\left(-\pi^{*} S\right)$ and $H F_{0}\left(-\pi^{*} S\right)$, where the grading comes from Maslov (or Morse) index (see [8]). Since $\mathcal{A}_{-\pi^{*} S}$ (and thus $S$ ) increases along the gradient lines (2), by choosing $x \in S^{-1}((\max S-\epsilon, \max S))$ for $\epsilon$ small 
enough, we see that if $\mu$ is a generator of $H F_{\operatorname{dim} M}\left(-\pi^{*} S\right)$, then

$$
\mu=k\left[x_{+}\right]+\sum_{x_{+} \neq x_{i} \in C F_{\operatorname{dim} M}} k_{i}\left[x_{i}\right]
$$

for some integers $k, k_{i}$, where $k \neq 0$. Assume that $\lambda<S\left(x_{+}\right)$. Then $x_{+} \notin$ $C F_{\text {dim } M}^{\lambda}\left(-\pi^{*} S\right)$, so that for every $a \in H F_{d i m M}^{\lambda}\left(-\pi^{*} S\right)$

$$
j_{*}^{\lambda}(a)=\sum_{x_{+} \neq x_{i} \in C F_{\operatorname{dim} M}} l_{i}\left[x_{i}\right] .
$$

Since $H F_{\operatorname{dim} M}^{\lambda}\left(-\pi^{*} S\right)$ is a free module with one generator $\mu$, it must be $j_{*}^{\lambda}(a)=c \mu$ for some integer $c$, i.e.

$$
c k\left[x_{+}\right]+\sum_{x_{+} \neq x_{i} \in C F_{\operatorname{dim} M}}\left(c k_{i}-l_{i}\right)\left[x_{i}\right]=0 .
$$

Since

$$
C F_{\operatorname{dim} M}\left(-\pi^{*} S\right) \ni c k x_{+}+\sum_{x_{+} \neq x_{i} \in C F_{\operatorname{dim} M}}\left(c k_{i}-l_{i}\right) x_{i} \notin \operatorname{Image}(\partial)
$$

and since $k \neq 0,(7)$ is possible only if $c=0$. Hence, $j_{*}^{\lambda}$ is not surjective. q.e.d.

\section{Flatness AND GEODESICS}

Denote by $\mathcal{F}(M)$ the space of normalized smooth functions on $M$ :

$$
\mathcal{F}(M):=\left\{S \in C^{\infty}(M) \mid \int_{M} S(q) d q=0\right\},
$$

where $d q$ is the Lebesgue measure induced by the Riemannian metric on $M$. Note that for every $C^{1}$-small Hamiltonian deformation $L$ of a zero section $O_{M}$ there is a unique $S \in \mathcal{F}(M)$ such that $L=\operatorname{Graph}(d S)$. Define a norm on $\mathcal{F}(M)$ by

$$
\|S\|:=\max _{q \in M} S-\min _{q \in M} S .
$$

Theorem 2. There exist $C^{1}$-neighborhoods $\mathcal{G}$ of $O_{M} \in \mathcal{L}$ and $\mathcal{U}$ of $0 \in \mathcal{F}(M)$ such that the mapping

$$
\Phi: \mathcal{U} \rightarrow \mathcal{G}, L \mapsto G \operatorname{raph}(d S)
$$

is an isometry.

Proof. $L:=\operatorname{Graph}(d S) \in \mathcal{G}$ is generated by the time-independent Hamiltonian $-\pi^{*} S$ (to simplify notation we keep the same notation for $-\pi^{*} S$ multiplied by a cut-off function). Hence $\rho(L)=\rho\left(\phi_{1}^{-\pi^{*} S}\left(O_{M}\right)\right)=\rho\left(-\pi^{*} S\right)$. It is easy to see that $\rho\left(\left(\phi_{1}^{-\pi^{*} S}\right)^{-1}\left(O_{M}\right)\right)=\rho\left(\pi^{*} S\right)$. Therefore, by Proposition 1

$$
\begin{aligned}
\max S-\min S & =\max S+\max (-S) \\
& =\rho\left(\pi^{*} S\right)+\rho\left(-\pi^{*} S\right) \\
& \leq d\left(O_{M}, L\right) \\
& \leq \int_{0}^{1}(\max S-\min S) d t \\
& =\max S-\min S .
\end{aligned}
$$


Hence, $d\left(O_{M}, L\right)=\|S\|$. The statement of the theorem follows from the invariance of $d$ :

$$
\begin{aligned}
& d\left(\operatorname{Graph}\left(d S_{0}\right), \operatorname{Graph}\left(d S_{1}\right)\right)=d\left(O_{M},\left(\phi_{1}^{-\pi^{*} S_{0}}\right)^{-1} \circ \phi^{-\pi^{*} S_{1}}\left(O_{M}\right)\right) \\
& =d\left(O_{M}, \phi_{1}^{\pi^{*} S_{0}-\pi^{*} S_{1}}\left(O_{M}\right)\right) \\
& =\quad d\left(O_{M}, \operatorname{Graph}\left(d\left(S_{1}-S_{0}\right)\right)\right) \\
& =\quad\left\|S_{1}-S_{0}\right\| \text {. }
\end{aligned}
$$

q.e.d.

Remark 3. Theorem 2 complements the conclusion made at the end of Section 7 of [7] that, if $c(\mu, L), c(1, L)$ are Viterbo's invariants of $L$ (see [10]), then $c(\mu, L)-$ $c(1, L) \leq d\left(O_{M}, L\right)$. From Theorem 2 it follows that the strict equality holds at least for $L C^{1}$-close to $O_{M}$.

Now we prove several statements analogous to the ones in [1] (or Section 5.7 in [4]).

Lemma 4 (Hamilton-Jacobi equation). Let $H(t, x)$ be a Hamiltonian generating $L_{t}=\operatorname{Graph}\left(d S_{t}\right)$. Then

$$
\frac{\partial S}{\partial t}(q)+H\left(t, d S_{t}(q)\right)=\text { const. }
$$

Proof. Let $q=\pi\left(\phi_{s}^{H}(y)\right)$ for $y \in M, s \in[0,1]$. Let $(q, p)$ be the canonical coordinates around $\phi_{s}^{H}(y)$. Denote $\left(q_{t}, p_{t}\right)=\phi_{t}^{H}(y)$ for $t$ near $s$. Then

$$
\left(q_{t}, p_{t}\right)=\left(q_{t}, \frac{\partial S}{\partial q}\left(q_{t}\right)\right)
$$

Differentiating with respect to $t$ we get

$$
\left(\frac{d q}{d t}, \frac{d p}{d t}\right)=\left(\frac{d q}{d t}, \frac{\partial^{2} S}{\partial t \partial q}+\frac{\partial^{2} S}{\partial q^{2}} \frac{d q}{d t}\right) .
$$

On the other hand, differentiating $H(t, d S(q)):=H\left(t,\left(q, \frac{\partial S}{\partial q}\right)\right)$ with respect to $q$ we obtain

$$
\frac{\partial}{\partial q}\left(H\left(t, q, \frac{\partial S}{\partial q}\right)\right)=\frac{\partial H}{\partial q}+\frac{\partial H}{\partial p} \frac{\partial^{2} S}{\partial q^{2}} .
$$

Applying Hamiltonian equations

to (8) and (9) we obtain

$$
\left\{\begin{array}{l}
\frac{d q}{d t}=\frac{\partial H}{\partial p} \\
\frac{d p}{d t}=-\frac{\partial H}{\partial q}
\end{array}\right.
$$

$$
\frac{\partial}{\partial q}\left(H(t, d S(q))+\frac{\partial S}{\partial t}(q)\right)=0
$$

q.e.d.

Corollary 5. If $H(t, x)$ is a Hamiltonian generating $L_{t}=\operatorname{Graph}\left(d S_{t}\right)$, then

$$
\left\|\frac{\partial S}{\partial t}\right\| \leq \max _{x \in T^{*} M} H(t, x)-\min _{x \in T^{*} M} H(t, x)
$$

for every $t$. 
Proof. By Lemma 4

$$
\begin{aligned}
\max _{x \in T^{*} M} H(t, x) & \geq \max _{q \in M} H\left(t, d S_{t}(q)\right) \\
& =c+\max _{q \in M}\left(-\frac{\partial S}{\partial t}(q)\right) \\
& =c-\min _{q \in M}\left(\frac{\partial S}{\partial t}(q)\right)
\end{aligned}
$$

and

$$
\begin{aligned}
\min _{x \in T^{*} M} H(t, x) & \leq \min _{q \in M} H\left(t, d S_{t}(q)\right) \\
& =c+\min _{q \in M}\left(-\frac{\partial S}{\partial t}(q)\right) \\
& =c-\max _{q \in M}\left(\frac{\partial S}{\partial t}(q)\right),
\end{aligned}
$$

i.e.

$$
-\min _{x \in T^{*} M} H(t, x) \geq-c+\max _{q \in M}\left(\frac{\partial S}{\partial t}(q)\right) .
$$

Adding (10) and (11) finishes the proof.

q.e.d.

Corollary 6 (Compare [1], Proposition 3.3.A). If $L_{t}=\operatorname{Graph}\left(d S_{t}\right)$, then $\frac{\partial S}{\partial t}$ is quasi-autonomous if and only if $\left\{L_{t}\right\}$ is generated by a quasi-autonomous Hamiltonian.

Proof. Note that $\left\{L_{t}\right\}$ is completely determined by values of $H(t, x)$ for $x$ near $\bigcup_{t} L_{t}$. Therefore, according to the comment after (2), we can assume that

$$
\max _{x} H(t, x)=\max _{x \in \cup_{t} L_{t}} H(t, x), \min _{x} H(t, x)=\min _{x \in \cup_{t} L_{t}} H(t, x) .
$$

Assume first that $\frac{\partial S}{\partial t}$ is quasi-autonomous, so that $\max _{q \in M} \frac{\partial S}{\partial t}=\frac{\partial S}{\partial t}\left(q_{+}\right)$and $\min _{q \in M} \frac{\partial S}{\partial t}=\frac{\partial S}{\partial t}\left(q_{-}\right)$. Then

$$
\begin{aligned}
0 & =\frac{\partial}{\partial q} \frac{\partial S}{\partial t}\left(q_{ \pm}\right) \\
& =\frac{\partial}{\partial t} \frac{\partial S}{\partial q}\left(q_{ \pm}\right)
\end{aligned}
$$

and thus $x_{\mp}:=d S_{t}\left(q_{ \pm}\right)$does not depend on $t$. Let $H_{t}$ be a Hamiltonian generating $\left\{L_{t}\right\}$. By Lemma 4 , for $x \in \bigcup_{t} L_{t}$

$$
\begin{aligned}
H(t, x) & =c-\frac{\partial S}{\partial t}(\pi(x)) \\
& \leq c-\frac{\partial S}{\partial t}\left(q_{-}\right) \\
& =H\left(t, x_{+}\right) .
\end{aligned}
$$

Similarly, $H(t, x) \geq H\left(t, x_{-}\right)$, i.e. $H$ is quasi-autonomous.

Assume now that $H(t, x)$ is a quasi-autonomous Hamiltonian, such that $\phi_{t}^{H}\left(O_{M}\right)$ $=L_{t}$. Let $\max _{x} H(t, x)=H\left(t, x_{+}\right)$and $\min _{x} H(t, x)=H\left(t, x_{-}\right)$. Again, we can 
assume that $x_{ \pm} \in \bigcup_{t} L_{t}$. Let $q_{ \pm}:=\pi\left(x_{\mp}\right)$. Then by Lemma 4

$$
\begin{aligned}
\frac{\partial S}{\partial t}(q) & =c-H\left(t, d S_{t}(q)\right) \\
& \leq c-H\left(t, x_{-}\right) \\
& =\frac{\partial S}{\partial t}\left(q_{+}\right) .
\end{aligned}
$$

Similarly, $\frac{\partial S}{\partial t}(q) \geq \frac{\partial S}{\partial t}\left(q_{-}\right)$, hence $S$ is quasi-autonomous.

q.e.d.

Remark 7. From the proof of Corollary 6 it follows that, if $H_{t}$ is a quasi-autonomous Hamiltonian generating $\operatorname{Graph}\left(d S_{t}\right)$, then (after modifying $H$ away from $\bigcup_{t} L_{t}$ if necessary)

$$
\max _{x \in T^{*} M} H(t, x)-\min _{x \in T^{*} M} H(t, x)=\max _{q \in M} \frac{\partial S}{\partial t}(q)-\min _{q \in M} \frac{\partial S}{\partial t}(q) .
$$

Theorem 8. A regular path $\left\{L_{t}\right\} \in \mathcal{L}$ is a geodesic if and only if it is generated by a locally quasi-autonomous Hamiltonian function.

Proof. Assume, without loss of generality, that $L_{0}=O_{M}$. Choose $\epsilon>0$ such that $L_{t} \in \mathcal{G}$ for $t \in(0, \epsilon)$. Let $S_{t}=\Phi^{-1}\left(L_{t}\right)$, where $\Phi$ is as in Theorem 2. $\left\{L_{t}\right\}_{0 \leq t \leq \epsilon}$ is a minimizing geodesic if and only if for every $\delta>0$ there exists a Hamiltonian $H$ such that $\phi_{t}^{H}\left(O_{M}\right)=L_{t}$ and

$$
\int_{0}^{\epsilon}\left(\max _{x} H(t, x)-\min _{x} H(t, x)\right) d t-\delta \leq d\left(O_{M}, L_{\epsilon}\right) .
$$

Since $\Phi$ is an isometry (Theorem 2)

$$
\begin{aligned}
d\left(O_{M}, L_{\epsilon}\right) & =\left\|S_{\epsilon}\right\| \\
& =\left\|\int_{0}^{\epsilon} \frac{\partial S}{\partial t} d t\right\| \\
& \leq \int_{0}^{\epsilon}\left\|\frac{\partial S}{\partial t}\right\| d t \\
& \leq \int_{0}^{\epsilon}\left(\max _{x} H(t, x)-\min _{x} H(t, x)\right) d t
\end{aligned}
$$

(the last inequality follows from Corollary 5 ). Since $\delta$ in (12) is arbitrary, it follows from (12) and (13) that

$$
\int_{0}^{\epsilon}\left\|\frac{\partial S}{\partial t}\right\| d t=\left\|\int_{0}^{\epsilon} \frac{\partial S}{\partial t} d t\right\|
$$

which according to (3) means that $\frac{\partial S}{\partial t}$ is quasi-autonomous. It follows from Corollary 6 that this is equivalent to $H(t, x)$ being quasi-autonomous. Vice versa, if $H$ (and thus $S$ as well) is quasi-autonomous, by (3) and Remark 7 both inequalities in (13) are equalities, and this gives

$$
d\left(O_{M}, L_{\epsilon}\right)=\int_{0}^{\epsilon}\left(\max _{x} H(t, x)-\min _{x} H(t, x)\right) d t .
$$

q.e.d.

Theorem 8 extends Theorem 1.3.D in $[1]$ which states that a regular path in a group $\operatorname{Ham}\left(\mathbf{R}^{2 n}\right)$ of compactly supported Hamiltonian diffeomorphisms of $\mathbf{R}^{2 n}$ is a geodesic if and only if it is generated by a locally quasi-autonomous Hamiltonian 
function. Indeed, a graph of every $\psi \in \operatorname{Ham}\left(\mathbf{R}^{2 n}\right)$ is a Hamiltonian deformation of a diagonal $\Delta \subset \mathbf{R}^{2 n} \times \mathbf{R}^{2 n}$. We can identify $\left(\mathbf{R}^{2 n} \times \mathbf{R}^{2 n}, d q \wedge d p-d Q \wedge d P\right)$ with $\left(T^{*} \Delta,-d(p d q)\right)$ through a symplectic identification

$$
(q, p, Q, P) \mapsto\left(\frac{q+Q}{2}, \frac{p+P}{2}, P-p, q-Q\right) .
$$

Since $\psi$ is compactly supported, the image of $\operatorname{Graph}(\psi)$ coincides with the zero section of $T^{*} M$ outside a compact set. Thus, after adding the fiber at infinity, $\operatorname{Graph}(\psi)$ can be considered as a Hamiltonian deformation of the zero section in $T^{*} \mathbf{S}^{2 n}$. Hence, Theorem $[2$ and Theorem 8 extend the analogous results in [1].

\section{REFERENCES}

[1] Bialy, M. and Polterovich, L., Geodesics of Hofer's metric on the group of Hamiltonian diffeomorphisms, Duke Math. J., 76 (1994), 273-292. MR 96b:58030

[2] Chekanov, Yu., Lagrangian intersections, symplectic energy, and areas of holomorphic curves, Duke Math. J., 95 (1998), 213-226. MR 99k:58034

[3] Floer, A., Cup-length estimates for Lagrangian intersections, Comm. Pure App. Math., 42 (1989), 335-356. MR 90g:58034

[4] Hofer, H., and Zehnder, E., Symplectic invariants and Hamiltonian dynamics, Birkhäuser Verlag, 1994. MR 96g:58001

[5] Lalonde, F., and McDuff, D., Hofer's $L^{\infty}$-geometry: energy and stability of Hamiltonian flows, I, II, Invent. Math., 122 (1995), 1-69. MR 96h:58063 errata MR 97b:58060

[6] Milinković, D., Morse homology for generating functions of Lagrangian submanifolds, Trans. AMS, 351 (1999), 3953-3974. MR 99m:58048

[7] Milinković, D., On equivalence of two constructions of invariants of Lagrangian submanifolds, Pacific J. Math., to appear.

[8] Oh, Y.-G., Symplectic topology as the geometry of action functional I - Relative Floer theory on the cotangent bundle, J. Diff. Geom., 45 (1997), 499-577. MR 99a:58032

[9] Oh, Y.-G., Symplectic topology as the geometry of action functional II - Pants product and cohomological invariants, Comm. Anal. Geom., 7 (1999), 1-55. MR 98a:58032

[10] Viterbo, C., Symplectic topology as the geometry of generating functions, Math. Ann., 292 (1992), 685-710. MR 93b:58058

Department of Mathematics, University of California, Irvine, California 92697-3875

E-mail address: dmilinko@math.uci.edu

Current address: Matematički Fakultet, Studentski trg 16, 11000 Belgrade, Serbia, Yugoslavia

E-mail address: milinko@matf.bg.ac.yu 